\title{
CCD detector performance for NOAO's wide-field MOSAIC cameras
}

Thomas Wolfe, Taft Armandroff, Morley M. Blouke, Travis Rector, Richard Reed, et al.

Thomas Wolfe, Taft Armandroff, Morley M. Blouke, Travis Rector, Richard Reed, Abhijit Saha, Robert Schommer, Chris Smith, Roger M. Smith, Alistair R. Walker, "CCD detector performance for NOAO's wide-field MOSAIC cameras," Proc. SPIE 3965, Sensors and Camera Systems for Scientific, Industrial, and Digital Photography Applications, (15 May 2000); doi: $10.1117 / 12.385427$

SPIE. Event: Electronic Imaging, 2000, San Jose, CA, United States 


\title{
CCD Detector Performance for NOAO's Wide Field MOSAIC Cameras
} Thomas Wolfe $^{a}$, Taft Armandroff ${ }^{a}$, Morley Blouke ${ }^{\mathrm{c}}$ Travis Rector ${ }^{\mathrm{a}}$, Rich Reed ${ }^{\mathrm{a}}$, Abhijit Saha ${ }^{\mathrm{a}}$, Robert Schommer ${ }^{\mathrm{b}}$, Chris Smith ${ }^{\mathrm{b}}$, Roger Smith ${ }^{\mathrm{b}}$ and Alistair Walker ${ }^{\mathrm{b}}$

\author{
${ }^{a}$ National Optical Astronomy Observatories, 950 North Cherry Avenue, Tucson, Arizona 85726 \\ ${ }^{\mathrm{b}}$ Cerro Tololo Inter-American Observatory, Casilla 603, La Serena, Chile ${ }^{\circ}$ Scientific Imaging \\ Technologies Incorporated, Beaverton, Oregon 97075
}

\begin{abstract}
In July of 1998 the National Optical Astronomy Observatories (NOAO ${ }^{1}$ ) successfully upgraded MOSAIC I, an 8192 by 8192 pixel array using eight Scientific Imaging Technologies, Inc. (SITe) ST-002A thinned backside $2 \mathrm{k}$ by $4 \mathrm{k}$ charge coupled devices (CCDs). In July of 1999 MOSAIC II, a clone of MOSAIC I was commissioned also using eight SITe ST-(0)2A CCDs. Additionally in December of 1998 NOAO implemented Mini-MOSAIC a 4096 by 4096 pixel array using two SITe ST-002A thinned CCDs. This report will discuss the performance, characterization and capabilities of the three wide field imagers now in operation at NOAO's Kitt Peak National Observatory (KPNO). Cerro Tololo Inter-American Observatory (CTIO) and at the WIYN Consortium 3.5-Meter telescope on Kitt Peak.
\end{abstract}

Keywords: CCD, CTIO, KPNO, MOSAIC, NOAO, SITe, ST002A, WIYN

\section{INTRODUCTION}

A decade ago NOAO started the design and development of CCD cameras to utilize the large field of view of their Astronomical Telescopes. Cameras using a MOSAIC of CCDs (Figure 1.) were designed and built for the Mayall 4-meter telescope at KPNO, the Blanco 4-meter telescope at CTIO and the 3.5-meter WIYN telescope on Kitt Peak. All of these cameras use the SITe $2 \mathrm{k}$ by $4 \mathrm{k}$ back illuminated three side buttable STO02A CCD as their detector. NOAO has tested and optimized the performance of twenty-one SITe ST002A CCDs. This paper will report on the performance of the STO02As that are in use in NOAO's two MOSAIC large field imagers and the WIYN Mini MOSAIC Camera. The characteristics reported on are the gain, read noise, charge transfer efficiency (CTE), full well, quantum efficiency (QE), dark current and measurements of the surface of the CCDs to verify a maximally flat imaging surface.

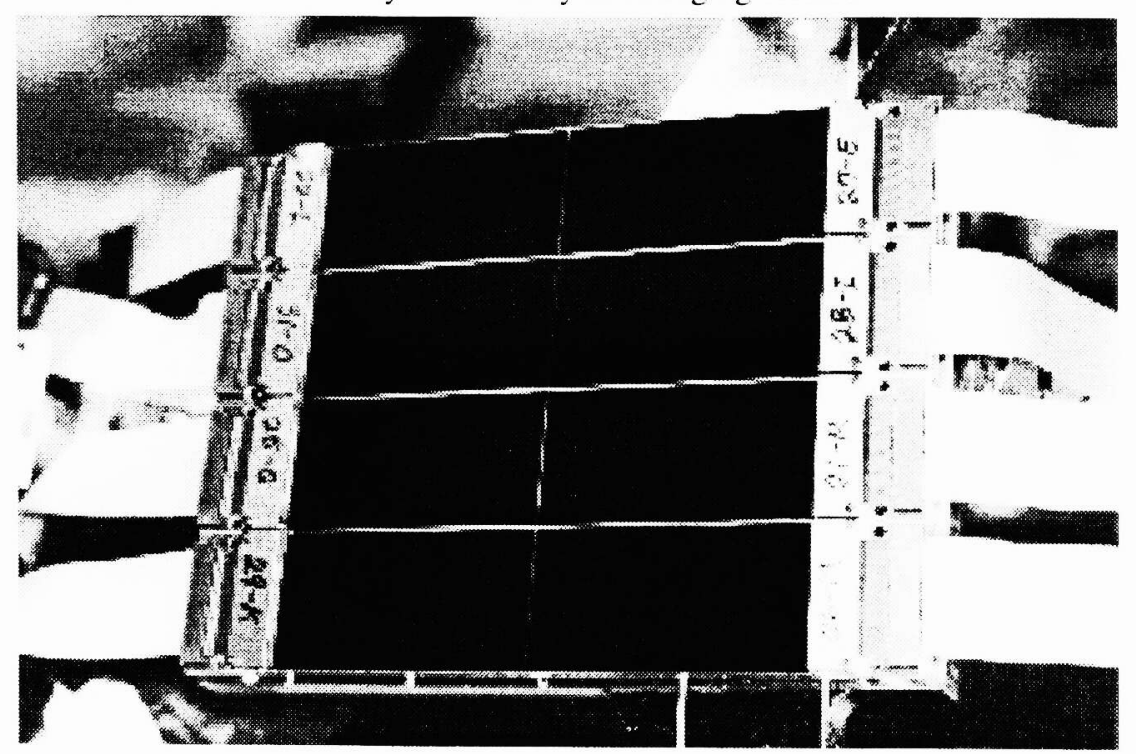

Figure 1 MOSAIC II Base Plate Mounted With Eight SITe ST002A CCDs

'National Optical Astronomy Observatories, operated by the Association of Universities for Research in Astronomy. Inc. (AURA) under cooperative agreement with the National Science Foundation. 


\section{CCD PERFORMANCE}

At NOAO initial test were performed on all the ST002A CCD. These tests characterized the performance of the CCDs and insure the CCD device meet specifications for NOAOs science grade cameras. The testing is done using a standard series of test at NOAO and the same set of test was performed on all the ST002A received. The CCDs are tested and specifications for gain, read noise, charge transfer efficiency, full well, quantum efficiency, and dark current are recorded. The testing is done and the same sets of test were performed on all the ST002A received for the large field imagers at NOAO. Voltages for all biases and clocks were initially set to the nominal voltages given to NOAO bye SITe (Table 1). These voltages are then adjusted if needed to optimize the performance of the CCDs for application in the wide field imagers. In the test system NOAO uses a video amplifier with a dual slope integrator and a 16 bit Analog to digital converter (ADC). For a detailed description of testing methods for NOAO's MOSAIC CCD cameras please read article number two listed in the references of this paper.

Table 1 Nominal CCD Clock Voltages for ST002A CCDs

\begin{tabular}{|c|c|c|c|}
\hline Gate & Voltage & Gate & Voltage \\
\hline $\begin{array}{l}\text { Voltage Diffusion Drain } \\
\text { (VDD) }\end{array}$ & +25.0 Volts & RESET GATES & 0 to +12 Volts \\
\hline $\begin{array}{l}\text { Voltage Reset Drain (VRD) } \\
\text { \& Voltage Overflow Drain } \\
\text { (OFD) }\end{array}$ & $\cdot+14.0$ Volts & SERIAL GATES & -6.0 to +5.0 Volts \\
\hline Last Gate & -4.0 Volts & SUMMING WELL & -5.0 to +5.0 Volts \\
\hline Substraight & 0.0 Volts & Parallel Gates 1,2 & -10.0 to +2.0 Volts \\
\hline $\begin{array}{l}\text { Package \& Amplifier } \\
\text { Ground }\end{array}$ & 0.0 Volts & $\begin{array}{c}\text { Parallel } 3 \text { Multi-Phased Pin } \\
\text { (MPP) }\end{array}$ & -7.5 to +6.0 Volts \\
\hline
\end{tabular}

To determine the gain and noise characteristics of the ST002As both the photon transfer technique and a technique that uses the radiation from an Iron-55 source were used. Iraf was used extensively for reduction of the DATA during the CCD testing phase of the MOSAIC projects. Test results for the SITe ST002As tested at NOAO are compiled in Table 2. Parameters for this table include amplifier sensitivity, system gain, read noise, full well, dark current, parallel charge transfer efficiency and serial charge transfer efficiency. Quantum efficiency (QE) testing is reported in Table 3, and shown graphically in figure 2. For additional information on NOAOs, CCDs and MOSAIC imagers refer to the articles listed in the references of this paper.

Flatness of the focal plane was important with these large field arrays of CCDs. At NOAO measurements of the surface of the CCDs were done to verify a flat imaging surface. The flatness of the CCDs as delivered from SITe did not meet the specifications required for the imagers so a shim with a complex and compound angle was fabricated uniquely for each CCD. Several iterations of measurements of the CCD plus shim and subsequent grinding of the shim were needed. This process was used to insure the desired specifications for the array focal plane flatness was achieved. Listed in Table 4 and Table 5 are the final measured surfaces of MOSAIC I and MOSAIC II respectively. For MOSAIC I the maximum height is 11,203 microns and the minimum height is 11,180 microns for a delta of 23 microns peak to valley. For MOSAIC II the maximum height is 11,203 microns and minimum height of 11,185 microns for a delta of 18 microns peak to valley. These values for flatness meet the scientific specification for flatness needed for NOAOs large field imagers. 


\begin{tabular}{|c|c|c|c|c|c|c|c|c|c|c|c|c|c|c|c|c|c|c|c|c|c|c|c|}
\hline \multicolumn{3}{|c|}{ Other } & \multicolumn{2}{|c|}{ Mini } & \multicolumn{8}{|c|}{ MOSAIC II } & \multicolumn{8}{|c|}{ MOSAIC I } & \multirow{2}{*}{ 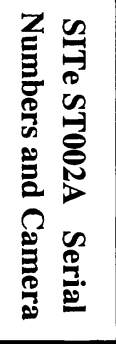 } & \multirow[b]{2}{*}{ Parameter } & \\
\hline & 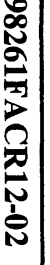 & 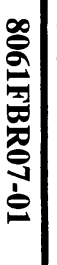 & & 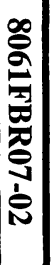 & & 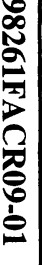 & 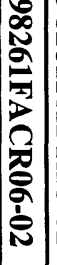 & 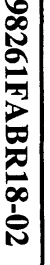 & 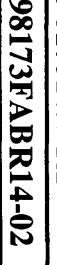 & 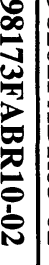 & 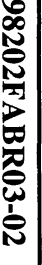 & 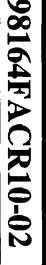 & 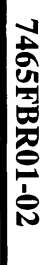 & 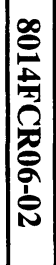 & 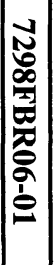 & 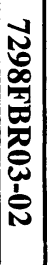 & 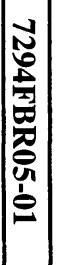 & 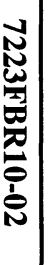 & 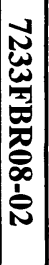 & 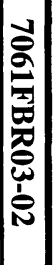 & & & \\
\hline $\overrightarrow{y_{3}}$ & $\vec{\Phi}$ & $\overrightarrow{\omega_{\infty}}$ & $\dot{0}$ & $\overline{\dot{v}_{n}}$ & $\overrightarrow{+}$ & $\bar{\Phi}$ & $\mid$\begin{tabular}{l|}
$\bar{u}$ \\
$\dot{u}$
\end{tabular} & $\vec{y}$ & \begin{tabular}{|l|}
$\overrightarrow{+}$ \\
$\dot{\infty}$ \\
\end{tabular} & $\vec{\sim}$ & $\vec{\omega}$ & $\vec{\omega}$ & $\overline{i_{\infty}}$ & $\vec{i}$ & \begin{tabular}{|l|} 
\\
$\dot{\infty}$ \\
\end{tabular} & \begin{tabular}{|l|} 
\\
$\infty$
\end{tabular} & 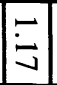 & $\overrightarrow{\dot{w}}$ & i & 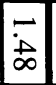 & & mplifier Sensitivity $\mu \mathrm{V} / \mathrm{e}^{-}$ & \\
\hline 잉 & $\begin{array}{l}0 \\
\infty \\
\infty\end{array}$ & $\dot{\mathrm{N}}$ & $\vec{\omega}$ & $\overline{\tilde{\omega}}$ & $\begin{array}{l}\circ \\
\otimes \\
\infty\end{array}$ & $\begin{array}{l}\circ \\
\infty \\
\infty\end{array}$ & \begin{tabular}{|l|} 
\\
\\
\end{tabular} & $\begin{array}{l}0 \\
8\end{array}$ & \begin{tabular}{l|} 
\\
$\dot{0}$ \\
$ن$ \\
$ن$
\end{tabular} \mid & 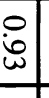 & $\overrightarrow{\tilde{u}_{n}}$ & $\dot{8}$ & i & \begin{tabular}{|l}
0 \\
0 \\
0 \\
0
\end{tabular} & $\div$ & $\bar{\omega}$ & $\overrightarrow{\tilde{\mathbf{o}}}$ & $\dot{8}$ & $\dot{9}$ & $\begin{array}{ll}\circ \\
\dot{0} \\
ن\end{array}$ & Syste & em Gain $15 \mu$ s Dwell ( $\left.\mathrm{e}^{-} / \mathrm{DN}\right)$ & \\
\hline$\stackrel{\substack{n \\
\oplus}}{n}$ & No & $\underset{i}{\omega}$ & $\vec{a}$ & $\overrightarrow{i n}$ & $\begin{array}{l}\omega \\
\Delta\end{array}$ & $\omega$ & $\left|\begin{array}{c}\omega \\
\Delta\end{array}\right|$ & $\underset{\sim}{\omega}$ & No & $\begin{array}{l}N \\
\infty\end{array}$ & \pm & 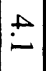 & $\stackrel{+}{\circ}$ & $\begin{array}{c}N \\
\infty\end{array}$ & $\begin{array}{l}\omega \\
\infty\end{array}$ & $\stackrel{\square}{\sim}$ & $\vec{\omega}$ & $\begin{array}{l}\omega \\
\sigma\end{array}$ & $\omega$ & $\left|\begin{array}{c}\omega \\
0\end{array}\right|$ & Systen & m Noise 15 $\mu$ s Dwell (e- RMS) & \\
\hline$\stackrel{\check{\partial}}{\circ}$ & $\dot{\omega}$ & $\dot{8}$ & $\begin{array}{l}n \\
\dot{2} \\
\end{array}$ & $\begin{array}{l}N \\
\dot{\omega} \\
\\
\end{array}$ & $\begin{array}{l}\dot{\infty} \\
\infty \\
\end{array}$ & $\vec{\omega}$ & $\dot{\tilde{0}}$ & ¿े & $\dot{\infty}$ & $\dot{N}$ & $\begin{array}{l}\sim \\
\dot{\omega} \\
\perp \\
\end{array}$ & 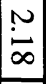 & i & $\underset{\infty}{\infty}$ & \begin{tabular}{|c|}
$N$ \\
8 \\
2
\end{tabular} & $\begin{array}{l}n \\
\vdots \\
a \\
\end{array}$ & \begin{tabular}{|c|}
$\tilde{N}$ \\
$\tilde{a}$ \\
\end{tabular} & $\overline{0}$ & \begin{tabular}{|l}
$N$ \\
0 \\
1 \\
\end{tabular} & $\left(\begin{array}{c}\dot{\infty} \\
\end{array}\right.$ & Syst & 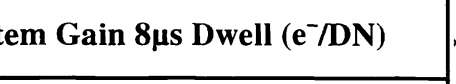 & \\
\hline$\ddot{v}$ & $\begin{array}{c}\omega \\
\Delta \\
\Delta\end{array}$ & $\vec{i}$ & $\left(\begin{array}{l}n \\
0\end{array}\right.$ & $\overrightarrow{0}$ & $\stackrel{+}{\oplus}$ & $\underset{\oplus}{\omega}$ & 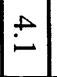 & $\overrightarrow{0}$ & $\overrightarrow{0}$ & $\underset{\infty}{\omega}$ & $\ddot{n}$ & 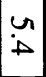 & $\overrightarrow{0}$ & $\vec{i}$ & $\underset{\infty}{+}$ & 9 & $\ddot{\omega} \mid$ & $\overrightarrow{i n}$ & $\overrightarrow{0}$ & $\stackrel{\leftrightarrow}{\oplus}$ & Syste & 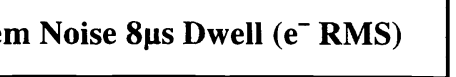 & \\
\hline $\begin{array}{l}\sim \\
\sim \\
0\end{array}$ & $\begin{array}{l}N \\
8\end{array}$ & \begin{tabular}{|c|c|}
$\omega$ \\
$\dot{\omega}$ \\
$u$
\end{tabular} \mid & \begin{tabular}{|l|}
$\vec{i}$ \\
$\dot{\tilde{y}}$
\end{tabular} & \begin{tabular}{|c}
$\omega$ \\
$\ddot{0}$
\end{tabular} & 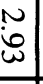 & $\begin{array}{l}n \\
\dot{\sigma} \\
\end{array}$ & \begin{tabular}{|c|}
$\sim$ \\
$\omega$ \\
$\omega$
\end{tabular} & $\stackrel{N}{*}$ & \begin{tabular}{|c|}
$N$ \\
$\infty$ \\
$N$ \\
\end{tabular} & $\stackrel{N}{\sim}$ & $\begin{array}{c}w \\
\infty \\
N \\
N\end{array}$ & $\begin{array}{l}\omega \\
\overrightarrow{1} \\
\infty\end{array}$ & $\begin{array}{l}\omega \\
\dot{\alpha}\end{array}$ & $\begin{array}{l}N \\
\infty \\
\infty \\
\end{array}$ & $\begin{array}{c}\omega \\
\omega \\
\omega\end{array}$ & $\begin{array}{l}\omega \\
\dot{\omega} \\
\end{array}$ & \begin{tabular}{|c|}
$\omega$ \\
$\dot{\omega}$
\end{tabular} \mid & $\begin{array}{l}w \\
a\end{array}$ & $\begin{array}{l}\omega \\
\dot{\omega} \\
\omega\end{array}$ & \begin{tabular}{|c}
$N$ \\
$\infty$ \\
- \\
-1
\end{tabular} & Syst & tem Gain $5 \mu \mathrm{s}$ Dwell $\left(\mathrm{e}^{-} / \mathrm{DN}\right)$ & \\
\hline 0 & $\ddot{0}$ & $\overrightarrow{i n}$ & $\infty$ & $\dot{\omega}$ & $\begin{array}{c}n \\
i \\
\end{array}$ & $u$ & $\ddot{n}$ & $\because$ & $\because$ & in & $\hat{a}$ & $a$ & $\ddot{v}$ & $\begin{array}{l}n \\
\dot{n}\end{array}$ & 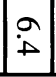 & $\dot{\omega}$ & $\begin{array}{l}\infty \\
\infty\end{array}$ & $\begin{array}{l}n \\
\infty \\
\infty\end{array}$ & 2 & $\begin{array}{l}n \\
\alpha\end{array}$ & Syste & m Noise $5 \mu$ s Dwell (e- RMS) & \\
\hline $\overrightarrow{ \pm}$ & $\begin{array}{l} \\
\dot{L} \\
\infty\end{array}$ & $\dot{\omega}$ & \begin{tabular}{|c|}
- \\
\end{tabular} & \begin{tabular}{|l|}
$\vec{\omega}$ \\
\end{tabular} & $\vec{\omega}$ & $\overrightarrow{\dot{u}_{n}}$ & \begin{tabular}{|l|l|}
$\dot{N}$ \\
\end{tabular} & $\bar{u}$ & \begin{tabular}{l|}
$\dot{\omega}$ \\
$\omega$
\end{tabular} & $\vec{\Delta}$ & $\vec{\nabla}$ & $\overrightarrow{\dot{a}}$ & $\overline{i_{\infty}}$ & $\overrightarrow{ \pm}$ & $\begin{array}{l} \\
\infty\end{array}$ & $\tilde{\tilde{N}}$ & ĩ & $\vec{i}$ & 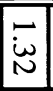 & $\dot{a}$ & & nplifier Sensitivity $\mathrm{mV} / \mathrm{e}^{-}$ & \\
\hline $\overrightarrow{8}$ & $\begin{array}{l}0 \\
\infty \\
0\end{array}$ & $\dot{\infty}$ & $\begin{array}{l}\dot{N} \\
\infty \\
\end{array}$ & $\begin{array}{l}\tilde{u}^{\prime} \\
\end{array}$ & $\begin{array}{l}\text { 인 } \\
\text { i } \\
\end{array}$ & $\begin{array}{l}0 \\
\infty \\
0 \\
\end{array}$ & $\left.\mid \begin{array}{l}0 \\
0 \\
0 \\
\end{array}\right]$ & $\begin{array}{l}0 \\
8 \\
\end{array}$ & \begin{tabular}{|l|} 
\\
i \\
N
\end{tabular} & $\begin{array}{l}0 \\
0 \\
0\end{array}$ & $\overline{\tilde{o}}$ & $\begin{array}{l}0 \\
\infty \\
\infty \\
+ \\
\end{array}$ & ì & $\begin{array}{l}0 \\
i \\
2\end{array}$ & $\dot{0}$ & $\dot{\bar{N}}$ & $\bar{\sigma}$ & 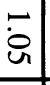 & $\dot{\ominus}$ & $\begin{array}{l}\circ \\
\infty \\
\circ \\
\end{array}$ & Syste & 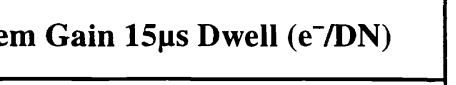 & \\
\hline $\begin{array}{l}\omega \\
\dot{\phi}\end{array}$ & $\omega$ & $\underset{\sim}{\omega}$ & $\stackrel{\oplus}{\sim}$ & $\overrightarrow{0}$ & $\begin{array}{l}N \\
\infty \\
\infty\end{array}$ & $\underset{\phi}{\omega}$ & $\omega$ & $\begin{array}{l}\omega \\
\dot{u}\end{array}$ & $\mid$\begin{tabular}{|l}
$\omega$ \\
$\omega$
\end{tabular} & 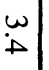 & $\begin{array}{c}\omega \\
0\end{array}$ & $\stackrel{N}{\sim}$ & 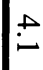 & $\omega$ & $\vec{\omega}$ & $\begin{array}{l}\omega \\
\alpha\end{array}$ & 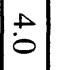 & $\begin{array}{l}\omega \\
\infty\end{array}$ & $\begin{array}{l}\omega \\
\omega\end{array}$ & $\dot{0}$ & Systen & m Noise $15 \mu \mathrm{s}$ Dwell ( $\left.\mathrm{e}^{-} \mathrm{RMS}\right)$ & \\
\hline $\overrightarrow{8}$ & $\begin{array}{l}5 \\
\infty\end{array}$ & \begin{tabular}{|l}
$N$ \\
$\dot{\omega}$ \\
\end{tabular} & $\begin{array}{l}N \\
\tilde{O} \\
\end{array}$ & \begin{tabular}{|l|} 
\\
$\dot{y}$ \\
\end{tabular} & $\dot{\omega}$ & के & $\vec{a}$ & 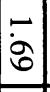 & $\dot{\omega}$ & $\dot{\infty}$ & $\stackrel{\sim}{\sim}$ & $\dot{\infty}$ & io & $\vec{\infty}$ & \begin{tabular}{|l}
$\mathbf{N}$ \\
$\mathbf{\omega}$ \\
\end{tabular} & $\begin{array}{c}N \\
0 \\
\end{array}$ & \begin{tabular}{|l|}
$N$ \\
$\infty$ \\
$\infty$
\end{tabular} & ஜे & $\begin{array}{l}N \\
0 \\
\end{array}$ & $\vec{a}$ & yst & em Gain 8 $\mu \mathrm{s}$ Dwell $\left(\mathrm{e}^{-} / \mathrm{DN}\right)$ & \\
\hline$\stackrel{+}{*}$ & $\vec{i}$ & $\overrightarrow{0}$ & in & $\ddot{a}$ & $\begin{array}{c}\omega \\
i \\
\end{array}$ & $\vec{i}$ & $+\infty$ & $\stackrel{+}{\oplus}$ & $+\vec{a}$ & $\stackrel{+}{\forall}$ & $\ddot{v}$ & $\stackrel{+}{\infty}$ & $\ddot{0}$ & $\vec{i}$ & $\underline{i}$ & $\ddot{1}$ & in & in & $\ddot{0}$ & $\underset{\omega}{\omega}$ & Syste & 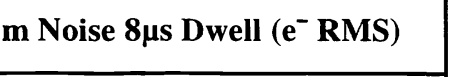 & \\
\hline$\omega$ & $\begin{array}{l}N \\
2 \\
\end{array}$ & \begin{tabular}{|c|}
$\omega$ \\
$\tilde{w}$ \\
\\
\end{tabular} & $\begin{array}{l}w \\
\dot{\delta}\end{array}$ & \begin{tabular}{|c|}
$\omega$ \\
$\dot{\delta}$ \\
$\dot{0}$
\end{tabular} & $\begin{array}{c}\sim \\
\infty \\
\infty \\
\end{array}$ & ra & \begin{tabular}{|l}
$N$ \\
$\hat{N}$
\end{tabular} & $\begin{array}{l}N \\
\dot{\alpha} \\
\end{array}$ & $\begin{array}{c}N \\
\mathrm{O}\end{array}$ & $\begin{array}{l}N \\
8 \\
8\end{array}$ & $\begin{array}{c}w \\
i \\
i\end{array}$ & $\begin{array}{l}N \\
\infty \\
\sim \\
\end{array}$ & $\begin{array}{l}\omega \\
\stackrel{\omega}{\Omega}\end{array}$ & $\begin{array}{l}n \\
\infty \\
0\end{array}$ & $\begin{array}{l}\omega \\
\dot{g}\end{array}$ & $\begin{array}{l}\omega \\
\dot{\omega} \\
\infty\end{array}$ & 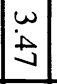 & $\begin{array}{l}\omega \\
\Xi \\
\Xi\end{array}$ & $\begin{array}{l}\omega \\
\dot{\sim} \\
\perp \\
\end{array}$ & $\begin{array}{c}n \\
\vdots \\
\vdots\end{array}$ & Syst & tem Gain 5 $\mu$ s Dell ( $\left.\mathrm{e}^{-} / \mathrm{DN}\right)$ & \\
\hline un & $\stackrel{\square}{\square}$ & in & $\infty$ & $\infty$ & $\vec{b}$ & $\stackrel{ \pm}{ \pm}$ & 0 & $\ddot{a}$ & $\begin{array}{l}n \\
\infty\end{array}$ & $\hat{a}$ & $\vec{\omega}$ & $\dot{i}$ & $\infty$ & $\infty$ & $\ddot{2}$ & in & $\hat{\omega}$ & 9 & $\vec{N}$ & $\ddot{n}$ & Syste & m Noise 5 $\mu$ s Dwell ( $\mathrm{e}^{-}$RMS) & \\
\hline$N$ & ఏి & 8 & 히 & $\infty$ & $\stackrel{\infty}{\infty}$ & ๘) & $|a|$ & ఏ & $\infty$ & $\stackrel{\infty}{N}$ & 8) & 8 & నิ & वे & 니 & $\infty$ & $\infty$ & $\vec{A}$ & $\overline{0}$ & $\vec{\omega}$ & & llwell @ 1\% Linearity $\left(\mathrm{Ke}^{-}\right)$ & \\
\hline- & - & - & $N$ & $N$ & - & - & - & - & - & - & - & - & - & - & - & - & - & - & $N$ & - & Dark & Current @ -90 ${ }^{\circ} \mathrm{C}\left(\mathrm{e}^{-} /\right.$pixel/hour $)$ & \\
\hline $\begin{array}{l}\circ \\
\text { : } \\
\text { : }\end{array}$ & $\begin{array}{l}0 \\
0 \\
0 \\
0 \\
0 \\
0 \\
0 \\
\infty \\
\end{array}$ & 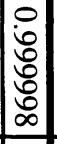 & 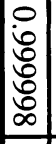 & 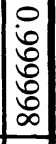 & $\begin{array}{l}0 \\
: \\
: \\
: \\
: \\
0 \\
\infty \\
\infty\end{array}$ & $\begin{array}{l}0 \\
\text { : } \\
8 \\
0 \\
0 \\
\infty\end{array}$ & $\mid \begin{array}{l}0 \\
8 \\
8 \\
8 \\
0 \\
0 \\
\infty\end{array}$ & 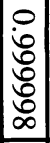 & $\mid \begin{array}{l}1 \\
\dot{8} \\
8 \\
: \\
0 \\
0 \\
\infty\end{array}$ & $\begin{array}{l}0 \\
8 \\
8 \\
: \\
0 \\
0 \\
\infty\end{array}$ & 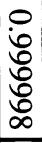 & $\begin{array}{l}0 \\
6 \\
0 \\
0 \\
8 \\
8 \\
\infty \\
\infty\end{array}$ & 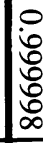 & $\begin{array}{l}0 \\
0 \\
0 \\
0 \\
0 \\
0 \\
\infty\end{array}$ & $\begin{array}{l}0 \\
\dot{0} \\
0 \\
0 \\
0 \\
0 \\
\infty\end{array}$ & $\begin{array}{l}0 \\
8 \\
8 \\
8 \\
8 \\
0 \\
0\end{array}$ & \begin{tabular}{|l|} 
\\
\\
8 \\
$:$ \\
0 \\
0 \\
0 \\
$\infty$
\end{tabular} & $\begin{array}{l}0 \\
i 0 \\
8 \\
0 \\
8 \\
0 \\
\infty \\
\infty\end{array}$ & $\begin{array}{l}0 \\
0 \\
8 \\
8 \\
8 \\
0 \\
\infty \\
\end{array}$ & \begin{tabular}{|l|} 
\\
$\dot{0}$ \\
8 \\
0 \\
0 \\
0
\end{tabular} & & CTE Parallel @ $1620 \mathrm{e}^{-}$ & \\
\hline O & \begin{tabular}{|l|}
0 \\
0 \\
8 \\
8 \\
8 \\
0 \\
$\infty$
\end{tabular} & 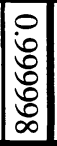 & 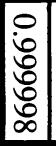 & \begin{tabular}{|l|} 
\\
\\
8 \\
8 \\
0 \\
0 \\
$\infty$
\end{tabular} & $\begin{array}{l}0 \\
: \\
: \\
: \\
0\end{array}$ & $\begin{array}{l}\text { 웅 } \\
\text { : } \\
\text { : }\end{array}$ & 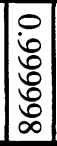 & io & \begin{tabular}{|l|} 
\\
\\
8 \\
0 \\
0 \\
0 \\
$\infty$
\end{tabular} & $\begin{array}{l}0 \\
8 \\
8 \\
8 \\
8 \\
\infty \\
\infty\end{array}$ & 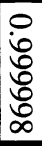 & 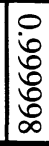 & $\begin{array}{l}0 \\
\text { i: } \\
\text { : }\end{array}$ & \begin{tabular}{|l} 
\\
0 \\
0 \\
0 \\
0 \\
0
\end{tabular} & 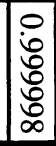 & $\begin{array}{l}0 \\
8 \\
8 \\
8 \\
8 \\
0 \\
\infty\end{array}$ & $\mid$\begin{tabular}{l|} 
\\
8 \\
8 \\
0 \\
0 \\
0 \\
$\infty$
\end{tabular} & 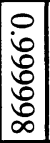 & $\begin{array}{l}0 \\
0 \\
8 \\
8 \\
8 \\
0 \\
0 \\
0\end{array}$ & $\begin{array}{l}1 \\
\text { : } \\
\text { : } \\
\text { : } \\
\infty\end{array}$ & & CTE Serial @ $1620 \mathrm{e}^{-}$ & \\
\hline
\end{tabular}

Table 2 CCD Test Results 


\begin{tabular}{|c|c|c|c|c|c|c|c|c|c|c|c|c|c|c|c|c|c|c|c|c|c|c|}
\hline \multicolumn{3}{|c|}{ Other } & \multicolumn{2}{|c|}{ Mini } & \multicolumn{8}{|c|}{ MOSAIC II } & \multicolumn{8}{|c|}{ MOSAIC I } & \multirow[b]{2}{*}{ 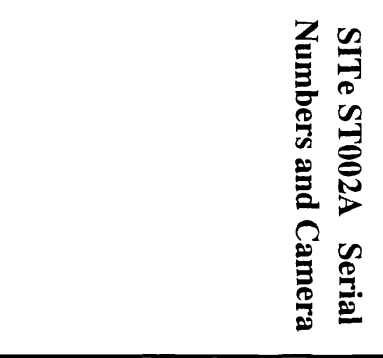 } & \\
\hline 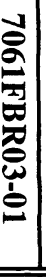 & 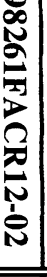 & 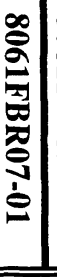 & 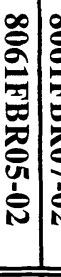 & 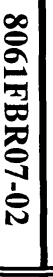 & 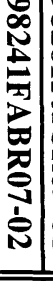 & $\begin{array}{l}0 \\
0 \\
0 \\
0 \\
0 \\
0 \\
0 \\
0 \\
0 \\
0 \\
0\end{array}$ & & 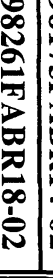 & 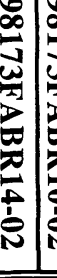 & 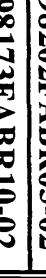 & 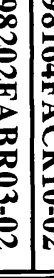 & & 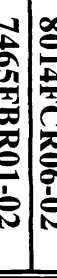 & 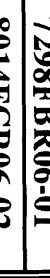 & 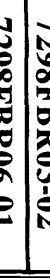 & 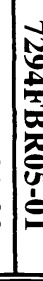 & 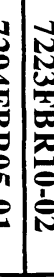 & 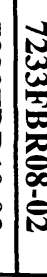 & 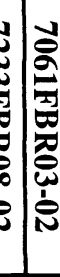 & & & \\
\hline نீ & $\stackrel{\infty}{\sim}$ & ตุ| & & & $\infty$ & $\stackrel{\infty}{v}$ & $\begin{array}{l}\infty \\
\sigma\end{array}$ & $\begin{array}{l}\infty \\
\stackrel{\bullet}{ }\end{array}$ & $\begin{array}{l}\infty \\
\sim\end{array}$ & $\stackrel{\infty}{*}$ & : & 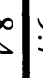 & \begin{tabular}{l|l}
0 \\
\end{tabular} & मे & 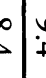 & $i$ & $\dot{\alpha}$ & 0 & ف) & $\begin{array}{l}0 \\
0\end{array}$ & $300 \mathrm{~nm}$ & \\
\hline $\begin{array}{l}\overrightarrow{0} \\
+\end{array}$ & $\begin{array}{l}\vec{\Delta} \\
\sigma\end{array}$ & $\vec{\infty}$ & \begin{tabular}{l|l}
$\infty$ & \\
$\omega$ & \\
\end{tabular} & $\begin{array}{l}\vec{\infty} \\
\text { in }\end{array}$ & $\vec{a}$ & $\overrightarrow{\vec{u}}$ & $\vec{i}$ & $\vec{a}$ & $\vec{\infty}$ & $\vec{\omega}$ & \begin{tabular}{l|l}
$\infty$ & \\
& \\
& \\
\end{tabular} & $\begin{array}{ll}\infty \\
\omega \\
\end{array}$ & \begin{tabular}{c|c}
$\infty$ & $\bar{b}$ \\
0 & $i$ \\
\end{tabular} & $\begin{array}{lll}0 & \\
0 & & \\
0\end{array}$ & \begin{tabular}{l|l}
$\infty$ & \\
$\Omega$ & \\
\end{tabular} & $\bar{\alpha}$ & \begin{tabular}{ll}
$\infty$ & 0 \\
\hdashline & 0
\end{tabular} & $\dot{a}$ & 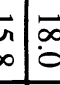 & $\begin{array}{l}\infty \\
0\end{array}$ & $320 \mathrm{~nm}$ & \\
\hline $\begin{array}{l}N \\
\sim \\
\rightarrow\end{array}$ & $\begin{array}{l}N \\
\infty \\
\infty\end{array}$ & స्र & & & $\begin{array}{l}\tilde{\omega} \\
\sigma \\
\sigma\end{array}$ & $\begin{array}{l}\stackrel{+}{+} \\
\triangleright \\
\end{array}$ & $\begin{array}{l}N \\
+ \\
\sigma\end{array}$ & $\begin{array}{c}w \\
\infty \\
\infty\end{array}$ & $\begin{array}{l}\sim \\
\sim \\
\sim\end{array}$ & $\stackrel{\sim}{\Delta}$ & $\begin{array}{c}\text { â. } \\
\text { Na }\end{array}$ & \begin{tabular}{l|l} 
\\
\end{tabular} & \begin{tabular}{c|c}
$N$ & 1 \\
0
\end{tabular} & S. & $\stackrel{1}{a}$ & 苛 & $\dot{c}$ & $\begin{array}{l}n \\
0 \\
0\end{array}$ & \begin{tabular}{l|l}
$ن$ & $\stackrel{N}{\sim}$ \\
\end{tabular} & U & $334 \mathrm{~nm}$ & \\
\hline$\frac{u}{0}$ & $\begin{array}{l} \pm \\
0\end{array}$ & $\stackrel{\sim}{\sim}$ & \begin{tabular}{l|l}
$\infty$ \\
$\infty$ \\
- & \\
\end{tabular} & $\begin{array}{l}\infty \\
\infty \\
u\end{array}$ & $\begin{array}{l}0 \\
0 \\
0 \\
0\end{array}$ & $\begin{array}{l} \pm \\
a\end{array}$ & $\begin{array}{l}\infty \\
i \\
i\end{array}$ & مे & $\begin{array}{l}0 \\
0 \\
0\end{array}$ & $\vec{a}$ & $\begin{array}{l}\text { y } \\
0 \\
\infty\end{array}$ & $\infty$ & 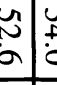 & 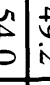 & \begin{tabular}{l}
$\overrightarrow{0}$ \\
\multirow{2}{*}{}
\end{tabular} & 品 & 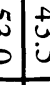 & $\begin{array}{l}\vec{t} \\
\stackrel{n}{*}\end{array}$ & \begin{tabular}{l|l}
$\vec{u}$ \\
$\overrightarrow{0}$ \\
\\
\end{tabular} & $\begin{array}{l}b \\
0 \\
\infty\end{array}$ & $365 \mathrm{~nm}$ & \\
\hline$\stackrel{8}{\circ}$ & $\begin{array}{l}1 \\
0 \\
\infty \\
\infty\end{array}$ & $\begin{array}{l}u \\
u \\
u\end{array}$ & 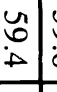 & $\begin{array}{l}n \\
b \\
a\end{array}$ & $\begin{array}{l}\mid \begin{array}{l}\mid \\
2 \\
\infty\end{array} \\
\end{array}$ & $\begin{array}{l}u \\
0 \\
a\end{array}$ & $\stackrel{8}{9}$ & $\begin{array}{l}4 \\
\infty \\
0 \\
0\end{array}$ & \begin{tabular}{l|l}
2 \\
$\infty$ \\
$\infty$
\end{tabular} & $\frac{0}{0}$ & 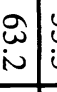 & & $\mathscr{2}$ & & \begin{tabular}{c|c}
$\infty$ & 0 \\
0 & 5 \\
\end{tabular} & & ב. & $\begin{array}{l}3 \\
\vdots\end{array}$ & 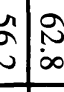 & $\begin{array}{l}2 \\
\infty \\
\end{array}$ & $380 \mathrm{~nm}$ & \\
\hline$\underset{\sim}{a}$ & $\begin{array}{l}2 \\
\infty \\
0\end{array}$ & $\stackrel{\infty}{-}$ & $\begin{array}{c}\text { Nิ) } \\
0 \\
0\end{array}$ & $\begin{array}{l}8 \\
0 \\
\end{array}$ & $\begin{array}{l}2 \\
\infty \\
\infty\end{array}$ & $\begin{array}{l}2 \\
\text { in }\end{array}$ & $\begin{array}{l}2 \\
\text { in }\end{array}$ & î & $\begin{array}{l}2 \\
\infty \\
\text { in }\end{array}$ & $\begin{array}{l}\infty \\
\infty \\
\dot{1} \\
\end{array}$ & $\begin{array}{l}\infty \\
\dot{\nu} \\
\end{array}$ & है & $\begin{array}{ccc}\infty & 1 \\
\infty & \vdots \\
\end{array}$ & $\begin{array}{c}U \\
\end{array}$ & $\vec{\Delta} \mid$ & مी & \begin{tabular}{l|l} 
\\
0 \\
0
\end{tabular} & $\begin{array}{lll} \\
b\end{array}$ & $\begin{array}{lll}1 & & \\
0 & \text { in } \\
\end{array}$ & $\begin{array}{l}3 \\
\text { un }\end{array}$ & $405 \mathrm{~nm}$ & \\
\hline $\begin{array}{l}\sim \\
\sim \\
\sim \\
\sim\end{array}$ & $\stackrel{\overrightarrow{1}}{+}$ & $\vec{w}$ & \begin{tabular}{l}
$a$ \\
$\infty$ \\
\hdashline
\end{tabular} & \begin{tabular}{|}
$\vec{\omega}$ \\
$\dot{\omega}$ \\
\end{tabular} & $\begin{array}{l}- \\
0 \\
\bullet \\
\end{array}$ & $\begin{array}{l}-1 \\
0 \\
\end{array}$ & $\begin{array}{l}N \\
\sigma \\
\end{array}$ & $\begin{array}{l}- \\
0 \\
\infty \\
\end{array}$ & $\begin{array}{l}\vec{u} \\
0 \\
0\end{array}$ & $\begin{array}{c}u \\
u \\
u\end{array}$ & $\underset{\infty}{\vec{*}}$ & ப. & \begin{tabular}{l|l}
$\Delta$ & $\vdots$ \\
$\perp$ & \\
\end{tabular} & \begin{tabular}{l|l}
$\infty$ & $\vdots$ \\
0 & \\
\end{tabular} & $\begin{array}{c}\mathbf{D} \\
\tilde{D}\end{array}$ & & $\begin{array}{l} \\
\end{array}$ & & $\vec{t}$ & $\begin{array}{l}\overrightarrow{1} \\
\vec{b}\end{array}$ & $450 \mathrm{~nm}$ & \\
\hline $\begin{array}{l}\text { जu } \\
\text { in }\end{array}$ & $\stackrel{\sim}{ \pm}$ & $\begin{array}{l}\overrightarrow{1} \\
a\end{array}$ & $\begin{array}{c}\vec{\omega} \\
\vec{\Delta} \\
\end{array}$ & $\begin{array}{l}- \\
\infty \\
u \\
\end{array}$ & $\begin{array}{l}1 \\
0 \\
0\end{array}$ & $\begin{array}{l}\text { जे } \\
\text { aे }\end{array}$ & $\begin{array}{l}\vec{u} \\
\omega\end{array}$ & $\begin{array}{l}\vec{u} \\
\Delta \\
\Delta\end{array}$ & $\begin{array}{l}\sim \\
\infty \\
u \\
u\end{array}$ & $\begin{array}{l}-1 \\
a \\
\end{array}$ & $\begin{array}{l}\vec{a} \\
\dot{\omega}\end{array}$ & $\vec{\Delta}$ & \begin{tabular}{l|l} 
जे & 9 \\
+ & + \\
\end{tabular} & \begin{tabular}{l|l}
$\infty$ & 3 \\
& \\
\end{tabular} & $\dot{0}$ & $\begin{array}{l}3 \\
\vdots\end{array}$ & ה. & 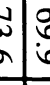 & \begin{tabular}{l|l}
0 \\
0 \\
0
\end{tabular} & $\begin{array}{l}4 \\
6 \\
\end{array}$ & $500 \mathrm{~nm}$ & \\
\hline $\begin{array}{l}\infty \\
\perp \\
\end{array}$ & \begin{tabular}{|l}
$\infty$ \\
$N$ \\
0 \\
\end{tabular} & $\stackrel{\infty}{\infty}$ & $\begin{array}{l}\vec{\Delta} \\
\vec{\Delta} \\
\end{array}$ & $\stackrel{\infty}{\sim}$ & $\begin{array}{l}\infty \\
\stackrel{\infty}{\sim} \\
\end{array}$ & 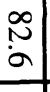 & \begin{tabular}{|l}
$\infty$ \\
$\sim$ \\
$\sim$ \\
\end{tabular} & $\begin{array}{l}\text { ज) } \\
\text { in }\end{array}$ & $\begin{array}{l}\infty \\
\omega \\
i \\
ن\end{array}$ & $\begin{array}{l}\infty \\
0 \\
0 \\
\end{array}$ & $\begin{array}{ll}\infty & \\
0 \\
ن \\
ن\end{array}$ & ปे & 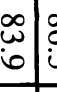 & 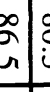 & $\begin{array}{lll}\infty & 0 \\
0 & \\
\end{array}$ & \begin{tabular}{lll}
0 & 0 \\
\hdashline & 0 \\
\end{tabular} & $\frac{\infty}{\infty}$ & $\begin{array}{l}j \\
j\end{array}$ & \begin{tabular}{c|c}
$\mathbf{u}$ & $\infty$ \\
$\omega$ & $\stackrel{\omega}{w}$ \\
\end{tabular} & $\stackrel{\infty}{\stackrel{\infty}{N}}$ & $550 \mathrm{~nm}$ & 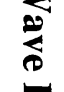 \\
\hline $\begin{array}{l}\infty \\
0 \\
0 \\
\end{array}$ & $\begin{array}{l}0 \\
0 \\
\sigma \\
\end{array}$ & \begin{tabular}{l|l}
$\infty$ \\
\hdashline \\
-
\end{tabular} & \begin{tabular}{l|l|}
$\infty$ \\
$\vdots$ \\
\end{tabular} & \begin{tabular}{|l|} 
\\
0 \\
0 \\
\end{tabular} & $\begin{array}{l}\infty \\
\leftrightarrow \\
\oplus \\
\end{array}$ & $\begin{array}{l} \\
2 \\
\omega \\
\end{array}$ & $\begin{array}{l}\infty \\
. \\
-\end{array}$ & \begin{tabular}{|l|}
$\infty$ \\
0 \\
0
\end{tabular} & \begin{tabular}{|l|}
$\infty$ \\
$\infty$ \\
$\oplus$ \\
\end{tabular} & $\begin{array}{l}\infty \\
\vdots \\
\omega\end{array}$ & \begin{tabular}{l|l}
$\infty$ \\
+ \\
$\infty$
\end{tabular} & $\begin{array}{l}\infty \\
0 \\
\\
\end{array}$ & \begin{tabular}{l|l}
$\infty$ & 0 \\
0 & 0 \\
0 &
\end{tabular} & \begin{tabular}{l|l}
8 & \\
0 & \\
\end{tabular} & \begin{tabular}{l}
$\infty$ \\
\hdashline
\end{tabular} & $\begin{array}{lll}0 & 0 \\
ن & \\
\end{array}$ & \begin{tabular}{l|l}
$\infty$ & 0 \\
$\infty$ & + \\
$\omega$ & + \\
\end{tabular} & $\begin{array}{lll}0 & \\
8 & \\
\end{array}$ & \begin{tabular}{l|l}
$\infty$ & $\infty$ \\
0 & $\infty$ \\
\end{tabular} & $\begin{array}{l}\infty \\
\infty \\
\infty\end{array}$ & $600 \mathrm{~nm}$ & 是 \\
\hline $\begin{array}{l}\infty \\
\infty \\
i\end{array}$ & $\begin{array}{l}\infty \\
\infty \\
\omega\end{array}$ & $\begin{array}{l}\infty \\
\infty \\
\infty\end{array}$ & $\begin{array}{l}\infty \\
\omega \\
\Delta \\
\end{array}$ & $\begin{array}{l}\infty \\
2 \\
6\end{array}$ & $\begin{array}{l}\infty \\
\infty \\
\infty\end{array}$ & \begin{tabular}{|l}
$\infty$ \\
$\omega$ \\
$\omega$
\end{tabular} & \begin{tabular}{|l}
$\infty$ \\
$\omega$ \\
$\omega$
\end{tabular} & 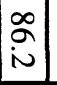 & $\begin{array}{l}\infty \\
\infty \\
\sigma \\
\sigma\end{array}$ & $\begin{array}{l}\infty \\
\infty \\
\infty\end{array}$ & \begin{tabular}{l}
$\infty$ \\
$\infty$ \\
\hdashline \\
\end{tabular} & $\infty$ & \begin{tabular}{l|l}
$\infty$ & \\
$\infty$ & \\
- & \\
\end{tabular} & 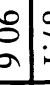 & $\stackrel{\infty}{\infty}$ & $\begin{array}{lll}\circ & 0 \\
0 & \vdots\end{array}$ & 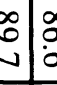 & 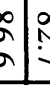 & $\begin{array}{lll}\infty & \infty \\
N & \infty & \infty \\
\end{array}$ & $\infty$ & $650 \mathrm{~nm}$ & \\
\hline $\begin{array}{l}\infty \\
u \\
\bullet \\
\end{array}$ & \begin{tabular}{l}
$\infty$ \\
$\propto$ \\
\hdashline \\
\end{tabular} & $\begin{array}{l}\infty \\
\sim \\
\omega\end{array}$ & 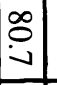 & $\begin{array}{l}\infty \\
+ \\
\infty\end{array}$ & $\left.\begin{array}{l}\infty \\
u \\
\infty\end{array}\right]$ & $\mid$\begin{tabular}{l}
$\infty$ \\
\hdashline \\
-1
\end{tabular} & $\begin{array}{l}\infty \\
2 \\
\omega\end{array}$ & $\stackrel{0}{\circ}$ & \begin{tabular}{|l}
$\infty$ \\
1 \\
$\omega$
\end{tabular} & $\begin{array}{c}\infty \\
i \\
\infty \\
\end{array}$ & 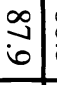 & 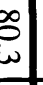 & \begin{tabular}{l|l}
$\infty$ \\
$\stackrel{2}{*}$ \\
\end{tabular} & $\begin{array}{l}\infty \\
\infty \\
\infty\end{array}$ & \begin{tabular}{c}
$\infty$ \\
$\vdots$ \\
\hdashline
\end{tabular} & $\begin{array}{l}0 \\
0 \\
0 . \\
\end{array}$ & \begin{tabular}{c|c}
$\infty$ \\
$\infty$ \\
$\infty$ & + \\
& + \\
\end{tabular} & \begin{tabular}{l|l}
0 & 8 \\
\hdashline & 1 \\
\end{tabular} & \begin{tabular}{l|l}
$\infty$ \\
$⿱ 亠 䒑 ⿻ 心 ㇒$ \\
$\infty$
\end{tabular} & $\begin{array}{l}\infty \\
+ \\
\dot{+} \\
\end{array}$ & $700 \mathrm{~nm}$ & \\
\hline 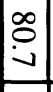 & $\infty$ & \begin{tabular}{|l}
3 \\
in
\end{tabular} & \begin{tabular}{|l|}
$\overrightarrow{3}$ \\
$\Delta$ \\
\end{tabular} & $\begin{array}{l}\vec{y} \\
\infty \\
\end{array}$ & $\begin{array}{l}-1 \\
0 \\
0\end{array}$ & \begin{tabular}{|}
$\sigma$ \\
$\infty$ \\
$\infty$
\end{tabular} & \begin{tabular}{|l|}
$\infty$ \\
$\infty$ \\
$\infty$ \\
\end{tabular} & $\begin{array}{l} \\
0 \\
0 \\
\end{array}$ & \begin{tabular}{|l|}
$\infty$ \\
0 \\
\hdashline \\
\end{tabular} & $\begin{array}{c}- \\
\infty \\
\infty\end{array}$ & $\stackrel{\infty}{\circ}$ & 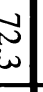 & \begin{tabular}{l|l} 
& \\
$\infty$ & \\
$\omega$ & \\
\end{tabular} & 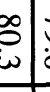 & 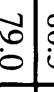 & $\begin{array}{l}\infty \\
0 \\
\end{array}$ & $\begin{array}{l}\infty \\
u\end{array}$ & 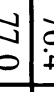 & \begin{tabular}{l|l}
$a$ & $\infty$ \\
$\dot{a}$ & $\infty$ \\
\end{tabular} & $\begin{array}{l}\infty \\
\infty \\
N \\
\end{array}$ & $750 \mathrm{~nm}$ & \\
\hline $\begin{array}{l}2 \\
0 \\
0\end{array}$ & $\begin{array}{l}2 \\
0 \\
\end{array}$ & $\begin{array}{l}\infty \\
- \\
-\end{array}$ & $\begin{array}{l}\infty \\
\infty \\
\infty \\
\infty\end{array}$ & $\begin{array}{l}2 \\
\infty \\
\\
\end{array}$ & $\begin{array}{l}8 \\
\text { in }\end{array}$ & \begin{tabular}{l}
2 \\
\hdashline \\
\end{tabular} & $\begin{array}{l}9 \\
\text { in } \\
\end{array}$ & $\begin{array}{l}0 \\
\text { in } \\
\text { in }\end{array}$ & 光 & $\begin{array}{l}9 \\
\text { in } \\
\end{array}$ & $\stackrel{\circ}{\circ}$ & 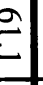 & $\begin{array}{ll}2 & \\
\infty & \\
\infty & \\
\end{array}$ & 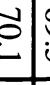 & $\begin{array}{ll}8 & \\
0 & \\
4\end{array}$ & \begin{tabular}{c|c}
0 \\
-15 \\
\end{tabular} & $\begin{array}{c}-2 \\
0\end{array}$ & \begin{tabular}{c|c}
$\infty$ \\
0 \\
0
\end{tabular} & $\begin{array}{lll}0 \\
0 \\
0\end{array}$ & $\begin{array}{l}\infty \\
\infty \\
+\end{array}$ & $800 \mathrm{~nm}$ & \\
\hline $\begin{array}{l}u \\
+ \\
\infty \\
\end{array}$ & $\begin{array}{l}n \\
\text { N } \\
\text { N }\end{array}$ & $\begin{array}{l}u \\
+ \\
0 \\
0\end{array}$ & $\begin{array}{l}u \\
\stackrel{1}{D} \\
\stackrel{D}{*}\end{array}$ & $\begin{array}{l}n \\
\infty \\
\infty\end{array}$ & $\begin{array}{l}n \\
0 \\
\end{array}$ & $\begin{array}{l}\tilde{n} \\
\dot{\omega} \\
\end{array}$ & $\begin{array}{c}\tilde{n} \\
\ddot{0} \\
\end{array}$ & $\left|\begin{array}{c}\tilde{} \\
2 \\
\omega\end{array}\right|$ & $\begin{array}{l}\text { un } \\
2 \\
\dot{\Delta}\end{array}$ & $\stackrel{u}{+}$ & $\begin{array}{l}n \\
\text { in }\end{array}$ & $\begin{array}{l}n \\
0 \\
0 \\
0\end{array}$ & $\begin{array}{l}n \\
\text { in } \\
\text { in }\end{array}$ & こू & ũ & $\begin{array}{lll}0 \\
0\end{array}$ & $\begin{array}{c}u \\
a \\
a\end{array}$ & $\begin{array}{c}n \\
\omega\end{array}$ & 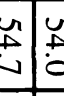 & $\begin{array}{l}n \\
\stackrel{1}{0} \\
0\end{array}$ & $850 \mathrm{~nm}$ & \\
\hline $\begin{array}{l}w \\
\infty \\
\infty\end{array}$ & $\begin{array}{l}\omega \\
- \\
-\end{array}$ & $\begin{array}{l}w \\
0 \\
0 \\
0\end{array}$ & $\begin{array}{l}\overrightarrow{0} \\
0 \\
\omega\end{array}$ & $\begin{array}{l}\overrightarrow{0} \\
\dot{\omega}\end{array}$ & $\stackrel{ \pm}{ \pm}$ & $\begin{array}{l}\omega \\
0 \\
\varnothing \\
\end{array}$ & \begin{tabular}{|c}
$w$ \\
$\infty$ \\
$\infty$ \\
\end{tabular} & \pm & $\begin{array}{l}\mathbf{N} \\
\mathbf{N} \\
\end{array}$ & 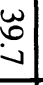 & $\stackrel{\oplus}{\sim}$ & $\begin{array}{l}\text { a } \\
\text { aे } \\
\end{array}$ & $\begin{array}{cc}0 \\
i \\
i\end{array}$ & $\begin{array}{l}b_{\infty} \\
\infty\end{array}$ & $\begin{array}{l} \pm \\
i\end{array}$ & 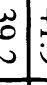 & $\stackrel{+}{0}$ & $\stackrel{+}{E}$ & 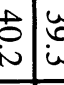 & $\begin{array}{l}\omega \\
\omega \\
\omega\end{array}$ & $900 \mathrm{~nm}$ & \\
\hline $\begin{array}{l}\sim \\
0 \\
0\end{array}$ & \begin{tabular}{|c}
$N$ \\
$\infty$ \\
$\infty$
\end{tabular} & $\begin{array}{c}\sim \\
\sim \\
\sim\end{array}$ & $\begin{array}{c}\tilde{1} \\
0 \\
0\end{array}$ & \begin{tabular}{|l}
$N$ \\
\\
in \\
\end{tabular} & $\begin{array}{l}\sim \\
\omega \\
\infty\end{array}$ & $\stackrel{N}{\perp}$ & $\begin{array}{l}N \\
\dot{A} \\
\text { in }\end{array}$ & $\begin{array}{l}\tilde{1} \\
0 \\
0\end{array}$ & $\mid \begin{array}{c}\sim \\
\sim \\
\sim\end{array}$ & $\begin{array}{c}n \\
\infty \\
\infty\end{array}$ & $\begin{array}{l}N \\
N \\
\text { N }\end{array}$ & $\begin{array}{c}\mathrm{N} \\
\mathrm{N} \\
\mathrm{N}\end{array}$ & $\begin{array}{l}\tilde{u} \\
\\
\end{array}$ & $\begin{array}{l}\tilde{2} \\
0 \\
0\end{array}$ & $\begin{array}{c}\tilde{N} \\
\tilde{N}\end{array}$ & $\begin{array}{l}\vec{a} \\
\end{array}$ & 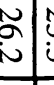 & $\tilde{u}$ & $\begin{array}{l}\tilde{u} \\
\dot{\sim}\end{array}$ & $\begin{array}{r}n \\
\vdots \\
i n\end{array}$ & $950 \mathrm{~nm}$ & \\
\hline$\Xi$ & $\mid \begin{array}{l}0 \\
0 \\
\alpha\end{array}$ & 供 & & & $\begin{array}{l}\bar{N} \\
0\end{array}$ & -1 & $\begin{array}{l}0 \\
\dot{2}\end{array}$ & $\bar{i}$ & 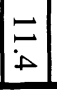 & $\bar{N}$ & \begin{tabular}{|l|l} 
\\
$\infty$ \\
$\infty$
\end{tabular} & $\Xi$ & $\bar{a}$ & $\Xi$ & $\begin{array}{lll} & \vec{\infty} \\
\infty & \\
\end{array}$ & 5 & \begin{tabular}{c|c}
$\vec{n}$ & $\hat{n}$ \\
0 & \\
\end{tabular} & $\vec{N}$ & $\begin{array}{ll}-\infty \\
\infty\end{array}$ & రి & $1000 \mathrm{~nm}$ & \\
\hline
\end{tabular}

Table 3 CCD Quantum Efficiency 


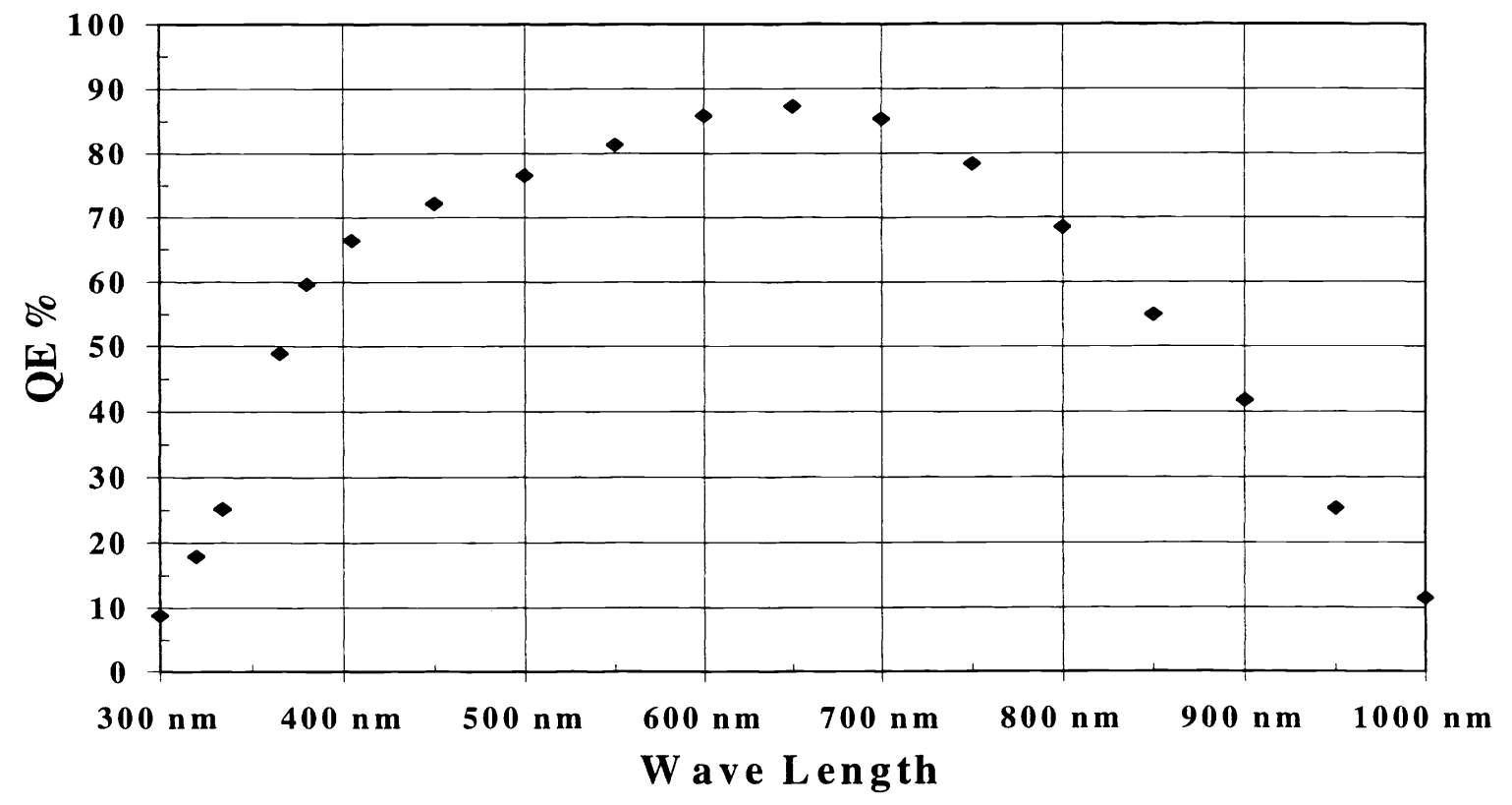

Figure 2 Average Quantum Efficiency NOAO's ST002A CCDs

\begin{tabular}{|c|c|c|c|c|}
\hline CCD Serial Number & Minimum Height & Maximum Height & Average Height & Delta \\
\hline 7061FBR03-02 & $11,188 \mu$ & $11,203 \mu$ & $11,195 \mu$ & $15 \mu$ \\
\hline 7233FBR08-02 & $11,191 \mu$ & $11,197 \mu$ & $11,195 \mu$ & $6 \mu$ \\
\hline 7223FBR10-02 & $11,193 \mu$ & $11,197 \mu$ & $11,195 \mu$ & $4 \mu$ \\
\hline 7294FBR05-01 & $11,188 \mu$ & $11,200 \mu$ & $11,196 \mu$ & $12 \mu$ \\
\hline 7298FBR03-02 & $11,194 \mu$ & $11,203 \mu$ & $11,200 \mu$ & $9 \mu$ \\
\hline 7298FBR06-01 & $11,194 \mu$ & $11,203 \mu$ & $11,199 \mu$ & $9 \mu$ \\
\hline 8014FCR06-02 & $11,194 \mu$ & $11,200 \mu$ & $11,197 \mu$ & $6 \mu$ \\
\hline 7465FBR01-02 & $11,180 \mu$ & $11,189 \mu$ & $11,186 \mu$ & $9 \mu$ \\
\hline
\end{tabular}

Table 4 Final Surface Measurements of CCD and Shim for MOSAIC I

\begin{tabular}{|c|c|c|c|c|}
\hline CCD Serial Number & Minimum Height & Maximum Height & Average Height & Delta \\
\hline 98164FACR10-02 & $11,188 \mu$ & $11,193 \mu$ & $11,191 \mu$ & $5 \mu$ \\
\hline 98202FABR03-02 & $11,185 \mu$ & $11,199 \mu$ & $11,193 \mu$ & $14 \mu$ \\
\hline 98173FABR10-02 & $11,194 \mu$ & $11,203 \mu$ & $11,199 \mu$ & $9 \mu$ \\
\hline 98173FABR14-02 & $11,187 \mu$ & $11,198 \mu$ & $11,194 \mu$ & $11 \mu$ \\
\hline 98261FABR18-02 & $11,191 \mu$ & $11,199 \mu$ & $11,195 \mu$ & $8 \mu$ \\
\hline 98261FACR06-02 & $11,192 \mu$ & $11,202 \mu$ & $11,197 \mu$ & $10 \mu$ \\
\hline 98261FACR09-01 & $11,193 \mu$ & $11,202 \mu$ & $11,197 \mu$ & $9 \mu$ \\
\hline 98241FABR07-02 & $11,186 \mu$ & $11,197 \mu$ & $11,193 \mu$ & $11 \mu$ \\
\hline
\end{tabular}

Table 5 Final Surface Measurements of CCD and Shim for MOSAIC II 


\section{NOAO'S MOSAIC I AND MOSAIC II PERFORMANCE}

NOAO's two 8192 by 8192 large field cameras are world class instruments. MOSAIC I, located at KPNO near Tucson, Arizona, is used at the 4-meter Mayall and 0.9-meter telescopes. MOSAIC II, located at CTIO in Chile, is used at the 4-meter Blanco telescope. The MOSAIC Imagers accommodate 8 SITe ST002A CCDs separated by a small gap. The CCDs have excellent cosmetic qualities and very high quantum efficiency. The sampling for the 4-meter class telescopes is 0.26 arc seconds per pixel and a field of view of 36 arc minutes. At the 0.9 -meter telescope the sampling is 0.43 arc seconds per pixel and a field of view of 59 arc minutes. Typical seeing is 0.6-0.7 arc seconds full width half max. The read out take approximately 150 seconds for the full $8 \mathrm{k}$ by $8 \mathrm{k}$ array and the read out is accomplished via four Arcon controllers. At the present time each Arcon reads out two CCDs through one amplifier on each CCD. Plans are underway to upgrade the Arcons to read the CCDs through two amplifiers per CCD and the read out time will be reduced to 100 seconds for the $8 \mathrm{k}$ by $8 \mathrm{k}$ array. The MOSAIC camera/dewar mounts inside the MOSAIC Instrument assembly. Raw images from the $8 \mathrm{k}$ by $8 \mathrm{k}$ MOSAIC are 135 Mbytes each and a typical night of approximately 70 images produces about 10 giga bytes of data. CCD characteristics can be found in Tables 4,5,and 6. For more information on NOAO's MOSAIC please refer to the articles listed in the reference of this paper.

Sample pictures from both MOSAIC I and MOSAIC II are displayed in Figure 3, Figure 4 and Figure 5 . Figure 3 is image of a raw, unprocessed Hydrogen alpha image of the Rosette taken with MOSAIC I $0.9 \mathrm{~m}$ telescope on Kitt Peak. Notice the distinct eight CCD outlined with a narrow gap. An overscan correction to bring each CCD bias down to the same level has been applied otherwise the image is unprocessed. Figure 4 is the same image of the Rosette Nebula (NGC 2237) but has been processed with false color. Five 10-minute exposures were made with Ha, O[III], and S[II] filters and assigned to the red, green, and blue color channels, respectively, to create this false color image. Figure 5 is a black and white image of the Sculptor Group galaxy NGC 247. The image was taken with the MOSAIC II camera and CTIO Blanco 4-m telescope on the night of Nov. 27, 1999. The large format of MOSAIC II combined with the large aperture of the Blanco telescope produced deep images and captured the entire galaxies and their surroundings in a single frame. Most of the fuzzy objects outside the main body of NGC 247 are distant background galaxies; some, however, are globular clusters native to NGC 247.

Both MOSAICs are very popular at NOAO and in the science community. This is demonstrated in the number of request for use of these instruments. The request statistics for observing semester 2000A (February 2000- July 2000) will be given. For this semester 146 nights requested to use MOSAIC I at the 4-meter at KPNO, 158 nights requested to use MOSAIC I at the 0.9-meter at KPNO and 98 nights were requested to use MOSAIC II at CTIO. The quality of the CCDs and the outstanding performance of the MOSAIC instruments make this a popular choice in the scientific community for large field imaging.

\section{MINI MOSAIC PERFORMANCE}

The Mini MOSAIC imager consists of two SITe ST002A 2k by 4k CCDs. It is configured into a 4096 by 4096 array with a small gap between the two CCDs. The CCDs have high quantum efficiency and have excellent cosmetic qualities. The imager has a resolution of 0.14 arc seconds per pixel and a large field of view at 9.6 arc minutes per side. Mini MOSAIC is used at the WIYN 3.5-meter telescope. Each of the two CCDs is read out via a Harcon controller and the four video outputs of the two CCDs is multiplexed through a single analog to digital converter. The read out time for this camera is approximately 182 seconds. CCD characteristics can be found in Tables 4,5, and 6 of this report

A sample image Figure 3 is included in the paper. This image of the globular cluster M3 (NGC 5272) was obtained with the Mini-MOSAIC camera on a Nasmyth focus of the WIYN 3.5m telescope on Kitt Peak. The white stripe down the middle of the image shows the gap between the active area of the two CCDs of the Mini MOSAIC camera. In this configuration, the pixel size is 0.14 arc seconds on the sky, and the field of view is 9.6 arc-minutes on a side. The image is shown with a logarithmic gray-scale. It is a 120-second exposure in the ' $\mathrm{B}$ ' passband, and images have full width at half maximum of better than 0.5 arc-seconds. Note the uniform image quality across the entire array. The inset shows how the superb seeing is very well sampled. The instrument thus exploits the superb seeing achieved by the WIYN telescope while also delivering uniform image quality over a moderately large field of view. 


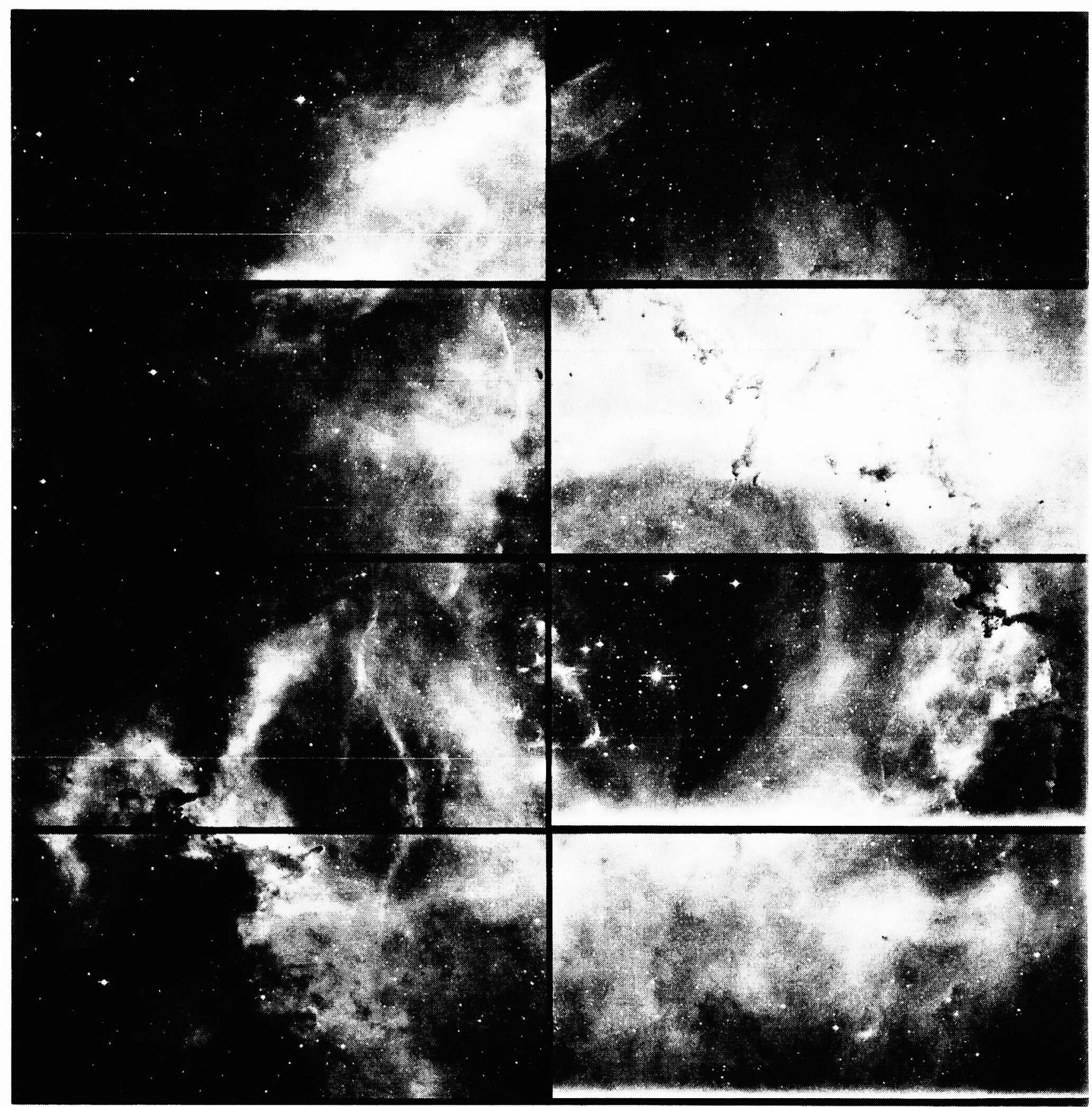

Figure 3 Image of a raw, unprocessed Hydrogen alpha image of the Rosette taken with MOSAIC I an array of eight SITe ST002A 2kx4k CCDs at the National Science Foundation's 0.9m telescope on Kitt Peak. An overscan correction to bring each $\mathrm{CCD}$ down to the same level has been applied otherwise the image is unprocessed.

Copyright Association of Universities for Research in Astronomy Inc. (AURA), all rights reserved National Optical Astronomy Observatories, operated by the Association of Universities for Research in Astronomy, Inc. (AURA) under cooperative agreement with the National Science Foundation. 


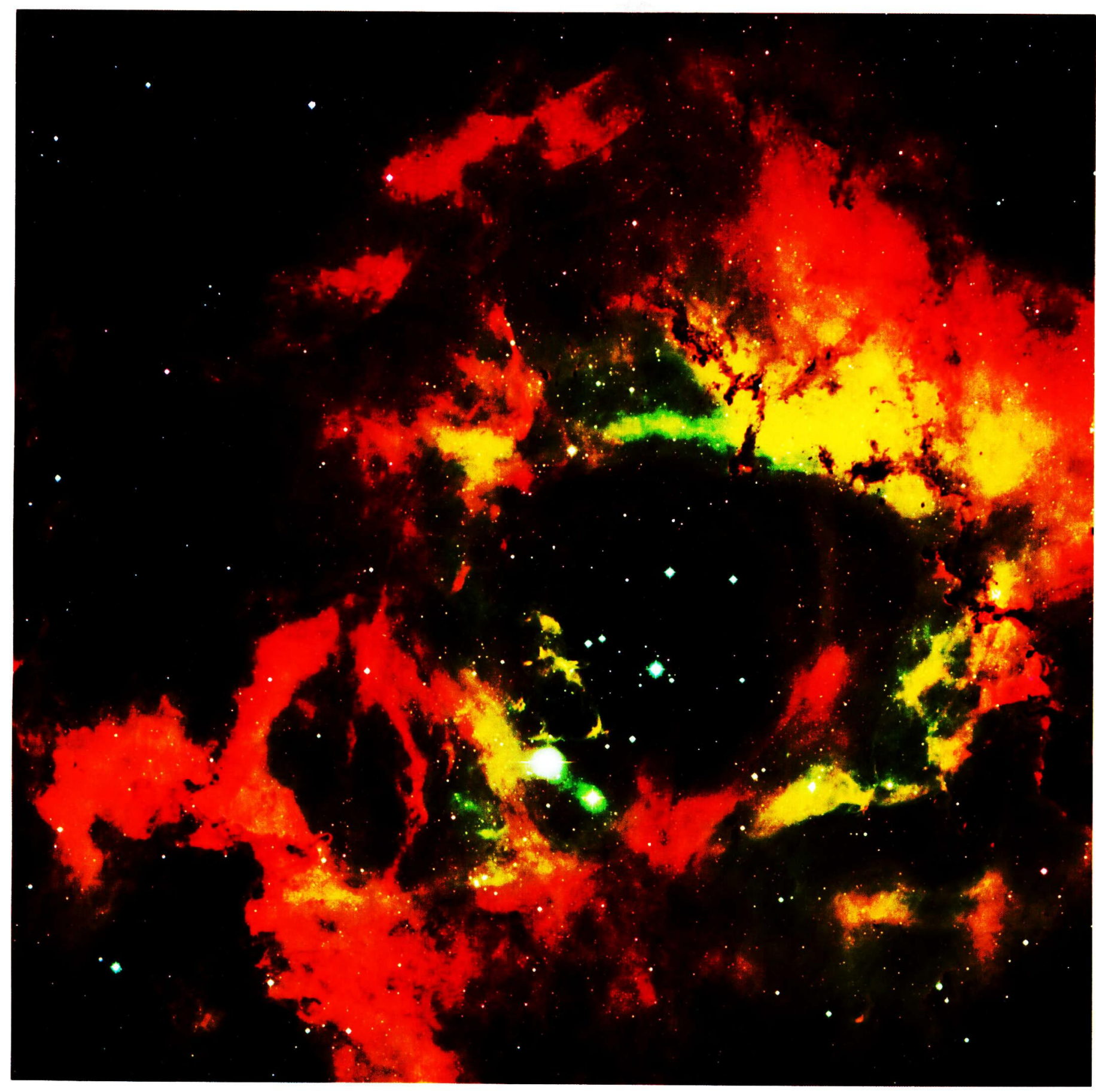

Figure 4 This image of the Rosette Nebula (NGC 2237) was taken with MOSAIC I an array of eight SITe ST002A $2 \mathrm{kx} 4 \mathrm{k}$ CCDs at the National Science Foundation's 0.9m telescope on Kitt Peak. Five 10-minute exposures were made with Ha (hydrogen), O[III] (oxygen), and S[II] (sulfur) filters and assigned to the red, green, and blue color channels, respectively, to create this false color image.

Copyright Association of Universities for Research in Astronomy Inc. (AURA), all rights reserved National Optical Astronomy Observatories, operated by the Association of Universities for Research in Astronomy, Inc. (AURA) under cooperative agreement with the National Science Foundation. 


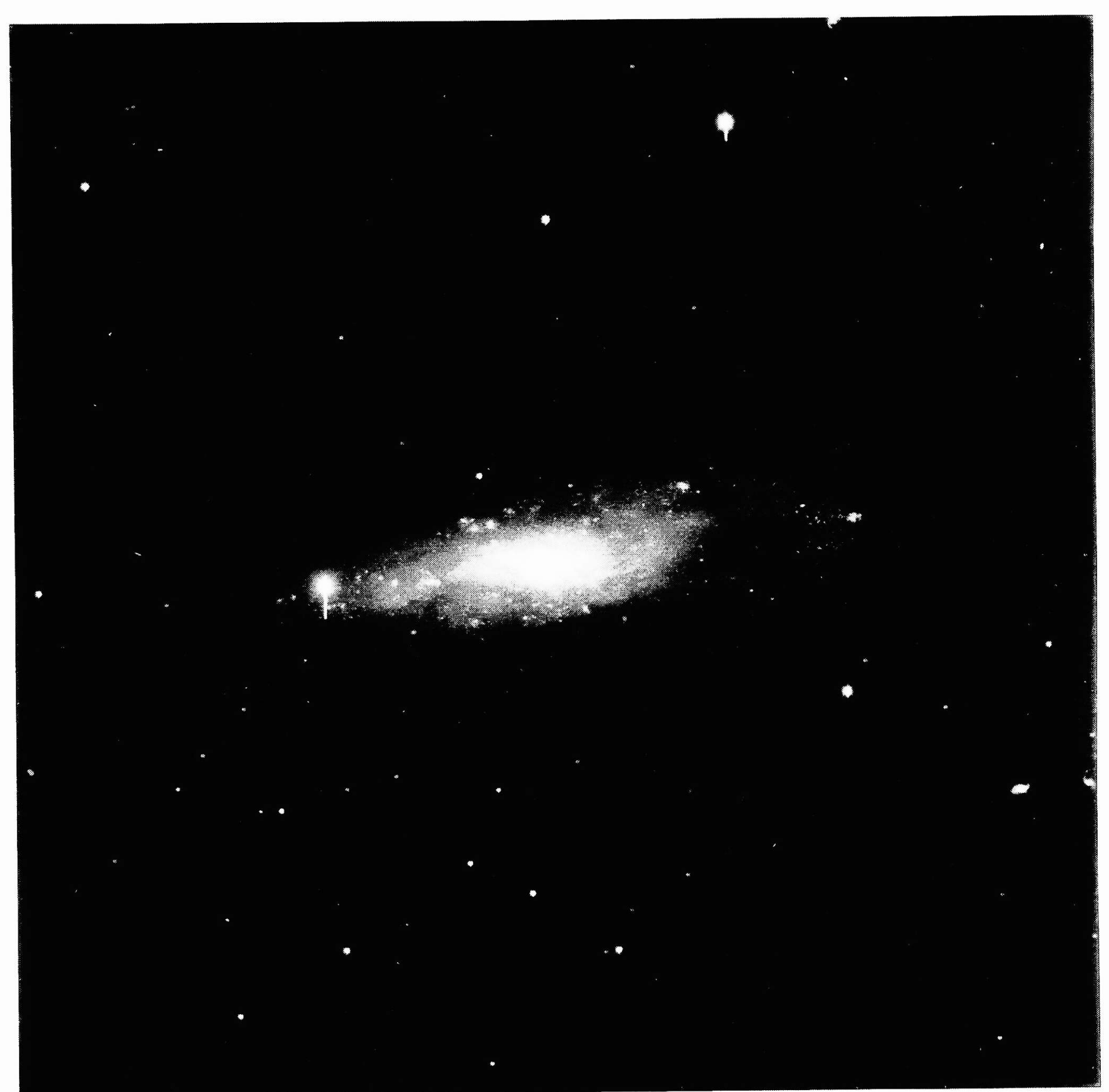

Figure 5 This image of the Sculptor Group galaxy NGC 247 was produced by combining frames taken through three different (blue, green, and red) filters. The images were taken with the MOSAIC II camera and CTIO Blanco 4-m telescope on the night of Nov. 27, 1999. The large format of MOSAIC II combined with the large aperture of the Blanco telescope produced deep images and captured the entire galaxies and their surroundings in a single frame. Most of the fuzzy objects outside the main body of NGC 247 are distant background galaxies; some, however, are globular clusters native to NGC 247. Astronomers Knut Olsen, Robert Schommer, and Nick Suntzeff (C'TIO) will use the images to identify and study the candidate globular clusters, the properties of which will provide clues as to how galaxies like NGC 247 formed. PHOTO CREDIT: K. Olsen, R. Schommer, N. Suntzeff,

Copyright Association of Universities for Research in Astronomy Inc. (AURA), all rights reserved National Optical Astronomy Observatories, operated by the Association of Universities for Research in Astronomy, Inc. (AURA) under cooperative agreement with the National Science Foundation. 


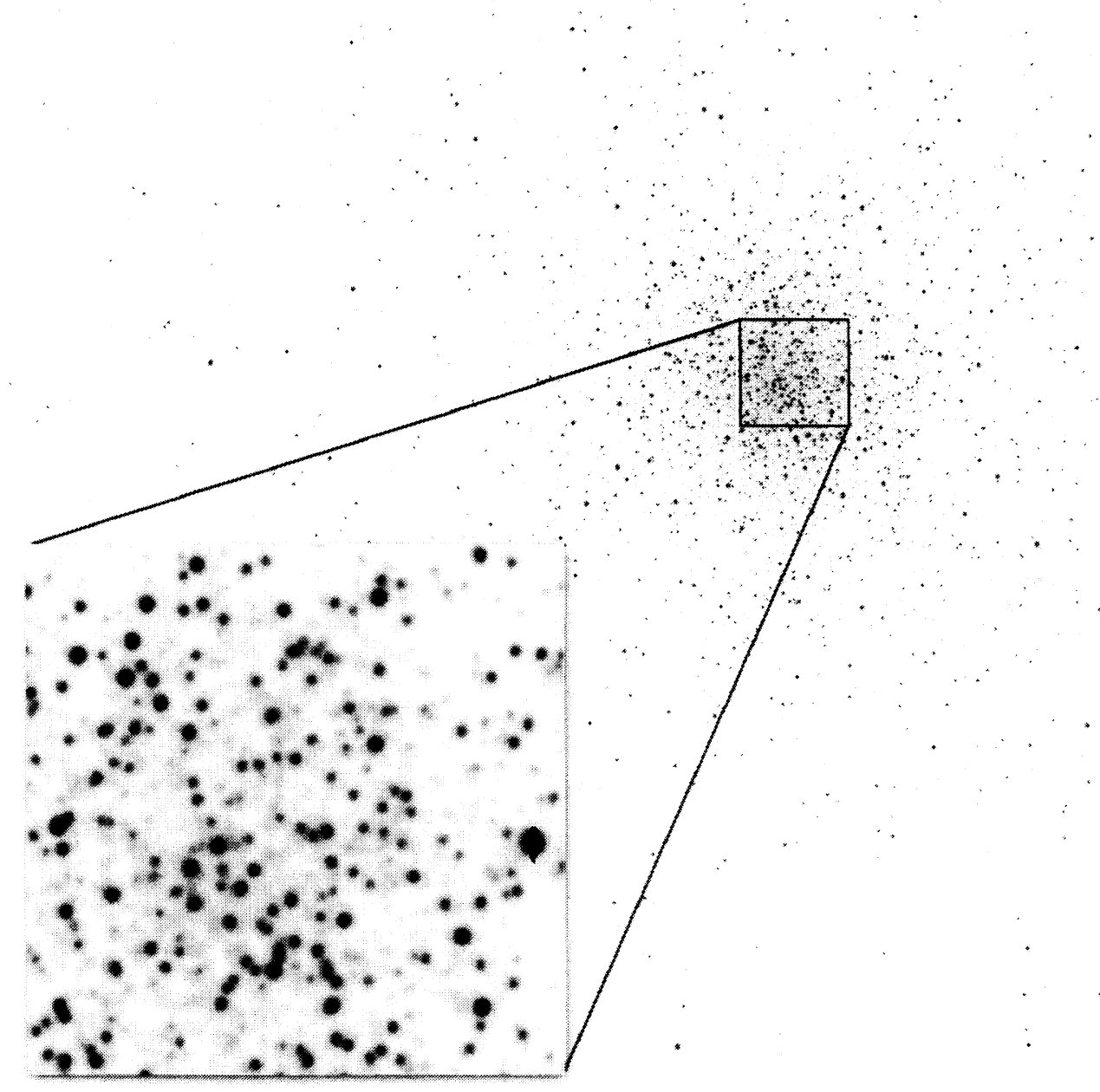

Figure 6 An image of the globular cluster M3 (NGC 5272) obtained with the Mini-MOSAIC camera on a Nasmyth focus of the WIYN 3.5m telescope on Kitt Peak. The camera uses two 2K X 4K SITE CCDs, mounted side by side for a $4 \mathrm{~K} \times 4 \mathrm{~K}$ field of view. The white stripe down the middle of the image shows the gap between the active area of the two CCDs of the Mini MOSAIC camera. In this configuration, the pixel size is 0.14 arc-seconds on the sky, and the field of view is 9.6 arc-minutes on a side. The image is shown with a logarithmic gray-scale. It is a 120-second exposure in the ' $B$ ' passband, and images have full width at half maximum of better than 0.5 arc-seconds. Note the uniform image quality across the entire array. The inset shows how the superb seeing is very well sampled. The instrument thus exploits the superb seeing achieved by the WIYN telescope while also delivering uniform image quality over a moderately large field of view.

Copyright WIYN Consortium, Inc., all rights reserved.

National Optical Astronomy Observatories, operated by the Association of Universities for Research in Astronomy, Inc. (AURA) under cooperative agreement with the National Science Foundation. 


\section{OTHER LARGE FORMAT DEVICES AT NOAO}

Other projects and proposals using large format devices are being developed at NOAO. At the WIYN telescope on Kitt Peak the WIYN Tip Tilt adaptive optics project will use a camera with a ST002A as the detector. NOAO facility in Chile is constructing a camera for the multi-fiber spectrograph HYDRA. This camera will also use a ST002A SITe device as its detector. Also at NOAO there is currently a study to determine the feasibility of a large survey telescope for KPNO. The instrument proposed for this telescope would include a camera that would have approximately 1000 CCDs.

\section{SUMMARY}

NOAO has developed Wide Field Imagers using detectors from Scientific Imaging Technologies, Inc. (SITe). The science grade ST-002A thinned backside illuminated $2 \mathrm{k}$ by $4 \mathrm{k}$, three side buttable charge coupled device (CCD) is the backbone of NOAO's large field cameras. The delivered performance in all of NOAOs wide field imagers is excellent. The outstanding qualities of these devices are the low read noise, the high quantum efficiency and low number of cosmetic defects. With these device a read noise of approximately 6 electrons RMS is attainable using double correlated sampling with 3microsecond dwell. A quantum efficiency of $86 \%$ peeks at $6000 \AA$ and these devices have response from $4,000 \AA$ to $10,000 \AA$. (This data based on an average for SITe ST-002A CCDs tested.) A comparative summary of the parameters of the three wide field imagers now in operation at NOAO is listed below in Table 6. With these wide field instruments NOAO will continue to provide outstanding science.

Table 6 Parameter Summary of NOAO's Large Field Imagers

\begin{tabular}{|c|c|c|c|}
\hline & MOSAIC I & MOSAIC II & Mini-MOSAIC \\
\hline Location & $\begin{array}{l}\text { KPNO 4-Meter Mayall Telescope; } \\
\text { KPNO 0.9-Meter Telescope }\end{array}$ & CTIO 4-Meter Blanco Telescope & WIYN Telescope Kitt Peak \\
\hline Arrays & $\begin{array}{c}8 \text { SITe ST002A 2048x4096 CCDs; } \\
\text { thinned science grade }\end{array}$ & $\begin{array}{c}8 \text { SITe ST002A 2048x4096 CCDs; } \\
\text { thinned science grade }\end{array}$ & $\begin{array}{l}\text { 2 SITe ST002A 2048x4096 CCDs; } \\
\text { thinned science grade }\end{array}$ \\
\hline Image Size & $\begin{array}{l}(8192 \times 8192)+\text { overscan }+ \text { header } \\
@ 16 \text { bits: } \sim 135 \text { Mbytes of Data }\end{array}$ & $\begin{array}{c}(8192 \times 8192)+\text { overscan }+ \text { header } \\
@ 16 \text { bits: } \sim 135 \text { Mbytes of Data }\end{array}$ & $\begin{array}{c}(4096 \times 4096)+\text { overscan }+ \text { header } \\
\text { @ } 16 \text { bits: } \sim 34 \text { Mbytes of Data }\end{array}$ \\
\hline Pixel Size & $\begin{array}{c}15 \mu \mathrm{m}(0.26 " / \text { picel @ 4-m; } \\
0.43^{\prime \prime} / \text { pixel @ 0.9-m) }\end{array}$ & $15 \mu \mathrm{m}(0.26 " /$ picel $)$ & $15 \mu \mathrm{m}(0.14 " /$ picel $)$ \\
\hline Gain & 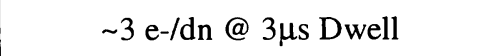 & 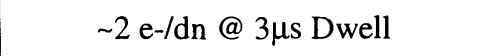 & 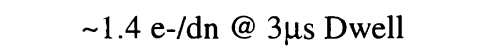 \\
\hline Read Noise & $\sim 6 \mathrm{e}-\mathrm{RMS}$ & $\sim 6 \mathrm{e}-\mathrm{RMS}$ & $\sim 6 \mathrm{e}-\mathrm{RMS}$ \\
\hline $\begin{array}{l}\text { Read out } \\
\text { Time }\end{array}$ & $\begin{array}{l}150 \text { Seconds in 8-channel mode; } \\
\text { (expected } \sim 100 \text { seconds in } 16- \\
\text { channel mode) }\end{array}$ & $\begin{array}{l}150 \text { Seconds in } 8 \text {-channel mode; } \\
\text { (expected } \sim 100 \text { seconds in } 16- \\
\text { channel mode) }\end{array}$ & $\begin{array}{l}182 \text { Seconds ( } 4 \text { video-amplifiers } \\
\text { multiplexed through one ADC) }\end{array}$ \\
\hline Saturation & $\begin{array}{l}\text { Typically, linear to } 0.1 \% \text { to } \\
70,000 \mathrm{e}-\end{array}$ & $\begin{array}{l}\text { Typically, linear to } 0.1 \% \text { to } \\
70,000 \mathrm{e} \text { - }\end{array}$ & $\begin{array}{l}\text { Typically, linear to } 0.1 \% \text { to } \\
70,000 \mathrm{e}-\end{array}$ \\
\hline $\begin{array}{l}\text { Quantum } \\
\text { Efficiency }\end{array}$ & 87\% peak@6000A & 87\% peak@6000Å & 84\% peak@6000Å \\
\hline $\begin{array}{c}\text { Dark } \\
\text { Current } \\
\end{array}$ & $\sim 2 \mathrm{e}-/$ pixel/hour & $\sim 2 \mathrm{e}-/ \mathrm{pixel} / \mathrm{hour}$ & $\sim 2 \mathrm{e}-/ \mathrm{pixel} / \mathrm{hour}$ \\
\hline $\begin{array}{l}\text { Field of } \\
\text { View }\end{array}$ & 36'x36'@4-m; 59'x59' @ 0.9-m & $36^{\prime} \times 36^{\prime}$ & $9.6^{\prime} \times 9.6^{\prime}$ \\
\hline
\end{tabular}




\section{ACKNOWLEDGEMENTS}

We would like to recognize all the Scientific Staff and Engineering Staff both in Tucson and La Serena, Chile. If it weren't for all the people involved pulling together and diligently working together as a team this report would not be possible. Thanks and kudos goes to more people than we can possible list here in this acknowledgment.

\section{REFERENCES}

1. Tood Boroson, Richard Reed, Woon-Yin Wong and Michael Lesser, "Development of a 8192 x 8192 CCD MOSAIC Imager" in Instrumentation in Astronomy VIII, D. Crawford and E. Craine, ed., Proc. SPIE 2189, pp. 877-885, March 1994.

2. Thomas Wolfe, Rich Reed, Morley Blouke, Todd Boroson, Taft Armandroff and George Jacoby, "CCD detector upgrade for NOAO's 8192 by 8192 MOSAIC”, in Optical Astronomical Instrumentation, S. D'Odorico, ed., Proc. SPIE 3355, pp. 487-496, March 1998.

3. F. G. Valdes and D. Tody, "The NOAO Data Handling System", in Optical Astronomical Instrumentation, S. D'Odorico, ed., Proc. SPIE 3355, pp. 487-506, March 1998.

4. Gary P. Muller, Rich Reed, Todd Boroson, Taft Armandroff and George Jacoby, "What is better than an $8192 \mathrm{X} 8192$ CCD MOSAIC Imager? Two MOSAIC Wide Field Imagers, one for KPNO and one for CTIO.”, in Optical Astronomical Instrumentation, S. D'Odorico, ed., Proc. SPIE 3355, pp. 577-585, March 1998.

5. http://www.noao.edu/

6. http://www.ctio.noao.edu/ctio.html

7. http://www.site-inc.com/

8. http://www.noao.edu/noao/pio/brochures/wiyn/text.html

9. http://www.aura-astronomy.org/

10. http://www.nsf.gov/

National Optical Astronomy Observatories, operated by the Association of Universities for Research in Astronomy, Inc. (AURA) under cooperative agreement with the National Science Foundation. 